\title{
New approaches for automated data processing of annually laminated sediments
}

\author{
I. $\operatorname{Rupf}^{1,{ }^{*}}$ and G. Radons ${ }^{1}$ \\ ${ }^{1}$ Institut für Physik der TU Chemnitz, Germany \\ *now at: Geological Survey of Baden-Württemberg, Freiburg i. Br., Germany
}

Received: 16 June 2004 - Revised: 15 November 2004 - Accepted: 17 November 2004 - Published: 24 November 2004

Part of Special Issue "Nonlinear analysis of multivariate geoscientific data - advanced methods, theory and application"

\begin{abstract}
Laminated sediments, like evaporites and biogenic lake sediments, provide high-resolution paleo-climate records. Yet detection and counting of laminae causes still problems because sedimentary structures are often disturbed. In the past laminated rocks often were analysed manually a tedious and subjective work.
\end{abstract}

The present study describes four automated approaches for lamina detection based on $1 \mathrm{~d}$ grey-scale vectors. Best results are obtained with a newly developed algorithm, called Adaptive Template Method (ATM) in combination with the Hilbert transform. ATM improves the signal to noise ratio of the grey-value signal. Its basic idea is to extract first a characteristic waveform, the template, which describes the typical grey-value variation transverse to the laminae. This is a kind of "template learning" process, which in practice is done by an appropriate averaging method. This template is in a second step used for processing the whole sample. One calculates the overlap of the template with the actual signal, the grey-value variation along the core, as function of position in core direction. This method generates a new signal with maxima at positions, where the template optimally matches the original signal. The new time-series is called ATtransform. It is smoother than the initial data sequence. High frequency noise and local trend effects are suppressed. Afterwards, the AT-transform can be analysed with the Hilbert transformation for extracting phase information.

The data processing methods are tested both on artificial data sequences and on a seasonally laminated sedimentary record of the Oligocene Baruth Maar (Germany). Although ATM is no panacea for highly disturbed signals, our comparison with other approaches shows that it provides the best results. The combination of ATM and the Hilbert transform allows to detect clearly long-term oscillations in the sedimentation patterns and thus cycles in climatic variations.

Correspondence to: I. Rupf

(rupf@lgrb.uni-freiburg.de)

\section{Introduction}

To study the climate of the past, high-resolution natural archives, so called climate proxies, are an important source of information. The highest-resolution climate data from ancient environments can be found in tree rings (e.g. Briffa et al., 1996), ice cores (Barnola et al., 1987), corals (Cole et al., 1992), and various types of annually laminated sediments (e.g. evaporitic, biogenic, and glacial meltwater lake sediments. For reviews see O'Sullivan, 1983; Anderson and Dean, 1988; and Glenn and Kelts; 1991). They are driven by several quasiperiodic and episodic forcing factors, like changes of solar irradiance (e.g. the 11 year sunspot cycle and multiple elements, as shown in Crowley et al., 1986; Bradbury and Dean, 1993; Dean et al., 2002; and Brauer et al., 1994), coupled ocean-atmosphere oscillations like ENSO (Cole et al., 1992) or NAO (White et al., 1996; Cook et al., 1998) and volcanic activity (e.g. Stuiver et al., 1995). All of these paleoclimate-proxies contain striped growth or sediment structures. Seasonally laminated organic lake deposits, so called varves, are of special interest because they reflect the development of temperature and precipitation in the midlatitudes. This study deals with the automated lamina detection and counting of this type of stratification. The processing of these patterns is still problematic because various sedimentary and postsedimentary processes often disturb rock structures. Although literature provides a large amount of studies on laminated sediments, the majority of these works use traditional manual or semi-manual methods for varve counting and thickness measurements. Common techniques are the analysis of thin sections under the microscope (Merkt and Müller, 1999; Brauer et al., 1999) or manually conducted investigations of scanned pictures (Dean et al., 2002). Besides the high amount of time-consuming and monotone labour, the main disadvantage of this class of methods is the high rate of subjectivity. Parallel varve counts of different researchers on the same data material of the Holzmaar (Eifel, Germany) differed by 5.8\% (Zolitschka, 1998). Francus et al. (2002) developed a semi-manual algorithm for 
varve counting. Although parts of the investigation process are automated, the method still contains highly subjective aspects of ordinary manual techniques.

There are only a few automated methods, described in literature. They are based on the analysis of scanned grey-value pictures of the laminated patterns. Grey-scale patterns transverse to the varves alternate periodically between higher and lower values, due to light and dark sublaminae.

D'Argenio et al. (1998) and Ripepe et al. (1991) work on $1 \mathrm{~d}$ grey-scale pixel lines. First, they filter the data by different approaches to diminish noise. Subsequently, Ripepe et al. (1991) removes bent and folded varve patterns by a geophysical method, called "Normal-moveout". Finally, these studies average several parallel grey-scale lines and detect lamina thicknesses. The main drawback of the methods is the high dependency of individual calculation steps on the specific object of interest. Every application on other data requires a high amount of algorithm tuning. The quality of the original data material and the scans have to be excellent. There should be no visible disturbances like core cracks, irregular layers, or postsedimentary alteration of sediment structures.

For future work, the method of Katsuta et al. (2003) seems to be most promising. Instead of treating single pixel lines, they use the full $2 \mathrm{~d}$ image for varve detecting. The aim of this method is the extraction of a sequential profile even from those laminations that show folded patterns and additional noise. To achieve this they differentiate grey-value matrices, calculate local slopes of laminations from the derivatives, eliminate noise and obtain a map of local slope lines. Subsequently, they are able to convert the map of folded slope lines to an unfolded representation and to compute an averaged $1 \mathrm{~d}$ profile of the corrected slope line map. But quality of the input data has to be even more excellent than in the techniques developed by Ripepe et al. (1991) or D'Argenio et al. (1998) because the calculation of local slope maps requires data sets without lateral and vertical disturbances. In using an ordinary $1 \mathrm{~d}$ method, irregularities due to inclined laminae can be avoided by shifting the vertical counting axis laterally to an undisturbed portion of the section. The Katsuta-algorithm does not provide this possibility. This prohibits application of this kind of technique in most of lacustrine laminites.

This study presents several approaches for automated lamina detection algorithms, based on $1 \mathrm{~d}$ grey-scale vectors of annually accumulated lake sediments. These methods are tested both on artificial data sequences and on seasonally laminated sedimentary records of the Oligocene Baruth Maar (Germany).

\section{Algorithms for the automated processing of laminites}

\subsection{Principles}

Annually laminated sediments contain several sublayers of different composition and colour. The simplest case is a yearly couplet, built up by a light and a dark lamina. Annu- ally changing accumulation rates of light and dark sediment layers lead to thickness variations of the varves. If thickness variations occur periodically, this phenomenon can be approximated as a phase modulated oscillation of the lengthdependent grey-scale signal with a base frequency of one year. The phase modulation probably depends on climatically driven causes. One simple model for this behaviour assumes the following spatial dependence of the grey-values:

$g s\left(x_{n}\right)=\sin \left(f_{C} 2 \pi x_{n}+B \sin \left(f_{M} 2 \pi x_{n}\right)\right)$.

$f_{C}$ is the so-called carrier or base frequency, $f_{M}$ the modulation frequency, and $B$ the modulation index (a measure for the ratio of the largest and smallest existing modulation frequency).

Grey-values are disturbed by various influences of sedimentary, post-sedimentary and technical processes. These effects complicate the automated detection of annual layers. In general, disturbances with time distortion, like badly scanned parts of pictures, can be distinguished from short-time irregularities (e.g. smaller core cracks or irregular siderite layers). The first category destroys information about inherent oscillations and therefore determines the boundaries of the individual data sequences. The second class can be eliminated without loss of information in time. In technical terms, disperse and visible disturbances should be separated. The actual extent of disperse and visible perturbations depends on image resolution. Disperse irregularities influence both amplitude and phase modulation. They can be partly eliminated by averaging over adjacent pixel rows. Visible disturbances, like irregular siderite layers, influence the normal alternation of grey-values considerably.

Automated methods are based on two different concepts. On the one hand, annual layer thicknesses can be extracted from the input signal. On the other hand, phase varying properties can be detected with the help of phase estimation methods based e.g. on concepts such as the Hilbert transform and the analytic signal. Unlike the first processing class, the second idea takes into account intra-annual events and oscillations. Certainly, some statistical properties of the layer distribution, such as thickness variations of light and dark layers, and correlation coefficients between them, cannot easily be detected with this second class of methods.

\subsection{Automated detection of lamina thickness variations}

The automated detection of light and dark layers from greyvalues is difficult. In the case of undisturbed data layerboundaries correspond to the inflexion points of the greyscale curves. But irregularities like intra-annual layers modify the shape of individual waveforms. Therefore, a previous smoothing of the data sequences is important. Normally, the grey-scale signal is nonstationary, so that local drift components should be removed before automated lamina-detection algorithms are applied. In the following, two methods will be introduced, using different smoothing- and detrending procedures. 


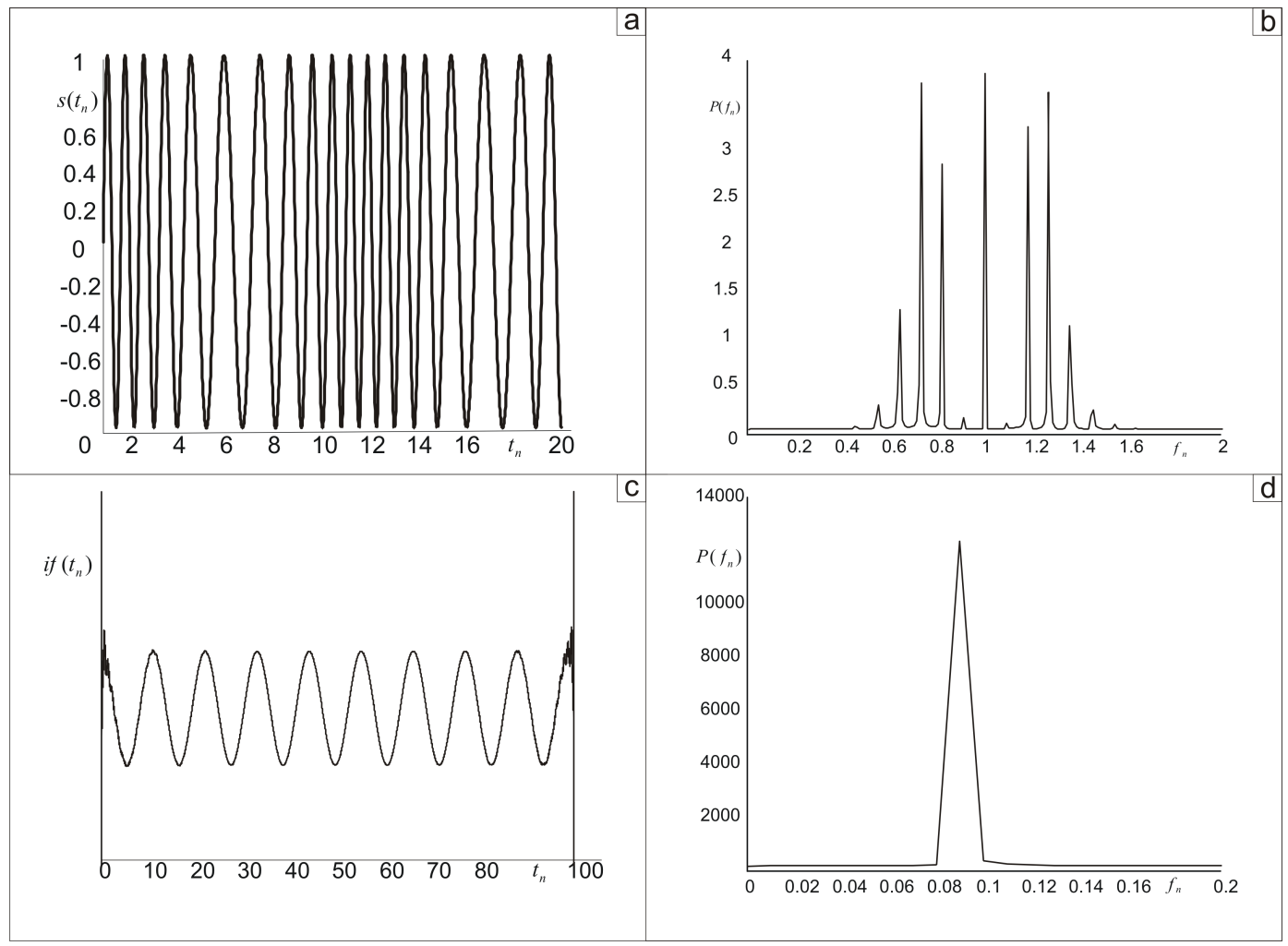

Fig. 1. (a) shows a phase modulated signal: $s\left(t_{n}\right)=\sin \left(2 \pi t_{n}+4 \sin \left(2 / 11 \pi t_{n}\right)\right)$ in time space, whereas (b) presents its power spectrum. Several peaks occur in the neighborhood of the carrier frequency peak. They are generated by the modulation process. After calculating the instantaneous frequency (c) from the analytic signal the corresponding periodogram exhibits one peak representing the modulation frequency (d).

\subsubsection{Differential Filters (DF)}

Differential Filters can be used for edge-detection, that means in terms of laminae the boundaries between light and dark layers (Cooper, 1997). Another positive effect is the reduction of local drift components.

$y\left(x_{n}\right)=D F\left(g s\left(x_{n}\right)\right)=g s\left(x_{n}\right)-g s\left(x_{n-1}\right)$.

The extrema of $y\left(x_{n}\right)$ correspond to the inflexion points of the grey-scale curve and indicate the boundaries between light and dark layers. This relationship can be used to calculate the thickness distributions of annual varves $t h\left(t_{n}\right)$, and their light and dark components $\left(t h_{l}\left(t_{n}\right), t h_{d}\left(t_{n}\right)\right)$.

\subsubsection{Moving Average Method (MAM)}

Like Differential Filters the moving average is a concept for the detection of boundaries between dark and light sublaminae. The main idea is to detrend the initial data sequence by computing the difference between the original data $g s\left(x_{n}\right)$ and a moving average $m\left(x_{n}, q\right)$ (Brockwell and Davis, 1991):

$m\left(x_{n}, q\right)=\frac{1}{2 q+1} \sum_{i=-q}^{q} g s\left(x_{n}+i\right)$, $w$ : window size of moving average $(w=2 q+1)$.

$y\left(x_{n}\right)=M A\left(g s\left(x_{n}\right)\right)=g s\left(x_{n}\right)-m\left(x_{n}, q\right)$.

The signal $y\left(x_{n}\right)$ has zeros at the boundaries between light and dark layers which can be used to compute lamina thickness variations.

\subsection{Automated detection of the instantaneous phase}

The Hilbert-transform is the fundamental method of the algorithm category, based on phase and frequency detection. One of the main problems of this type of automated procedures is the occurrence of highly disturbed signals, so that instantaneous properties like the phase information cannot be detected anymore. Therefore, it advisable to filter signals before applying a phase detection method. Natural data sets of laminated sediments are often very noisy, so that the corresponding periodograms do not show a well-defined peak at the one year base frequency. In addition intra-annual events and years of low sedimentation rates generate periodogram peaks in the same frequency range. These facts complicate the usage of traditional methods of data preprocessing like band-pass filters. Tests with simple band-pass filters, which we applied to our real data sets before applying the Hilbert transform, were not very successful. 


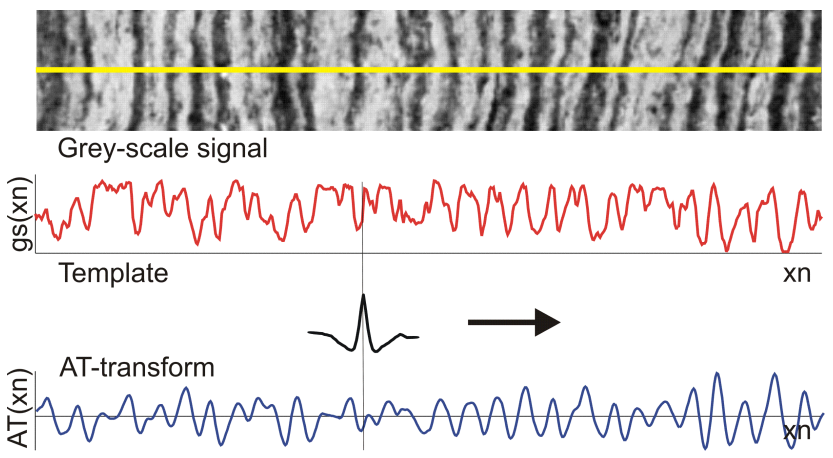

Fig. 2. A typical scanned picture of annually laminated lake sediments of the Upper Oligocene Baruth Maar and its corresponding grey-scale diagram. The template was computed with ATM. The AT-transform (the scalar product between the grey-scale signal and the template) is smoother than the initial signal. High frequency noise and local drift components are suppressed.

Alternative methods for phase estimation were used in DeShazer et al. (2001) and Quian et al. (2002) in the context of synchronisation measures. DeShazer et al. (2001) define a so called Gaussian phase, which is basically the phase of the Gabor transform of a real signal computed at some fixed frequency. Similarly Quian et al. (2002) generate a complex signal by convolving the real time series with a complex Morlet wavelet of distinct central frequency and width for calculating phase properties. Thus both approaches try to extract the phase at a certain predefined frequency. If the one year base frequency and the range of frequency modulation is well defined, such algorithms provide interesting options. But as mentioned above natural data sets such as ours do not have this property. We also calculated the Gabor transform of our data sets and found that the phase of the transformed signal depends sensitively on the frequency argument of the Gabor transform. Therefore for our data this is not a well suited method for determining the phase. We attribute this to the fact that similar to the method of bandpass filtering a fixed frequency window is used, which does not exploit the inherent structures of the signal. Therefore, a special filtering-method, which uses inherent data structures and which is based on the wavelet idea (Adaptive Template Method), was developed. It will be applied to the grey-scale data before using the Hilbert transform.

\subsubsection{Hilbert transform and analytic signals (HT)}

The analytic signal $z(t)$ as a part of the concept of the Hilbert transform is a useful tool for the detection of time varying signal attributes. It was first introduced by Gabor (1946). The analytic signal is defined as:

$z(t)=x(t)+i \tilde{x}(t)$

$x(t)$ is the original real signal, $\tilde{x}(t)$ represents the Hilbert transform. In the time domain the Hilbert transform can be expressed as

$\tilde{x}(t)=\frac{1}{\pi} p v\left\{\int_{-\infty}^{+\infty} \frac{x(\tau)}{t-\tau} d \tau\right\}$,

where $p v$ is the Cauchy principle value (Pikovski et al., 2001).

Original signal and Hilbert transform differ by a phase delay of $90^{\circ}$ in each frequency component. Therefore the Hilbert transform of a pure sine wave is a negative cosine. The analytic signal has several important temporal properties, which can be used to detect amplitude, phase or frequency information (Cramèr and Leadbetter, 1964). It can be regarded as an anticlockwise rotating vector in a complex plane (Mari et al., 1999). The time-varying angular velocity is also called instantaneous phase $\varpi(t)$ :

$\varpi(t)=\arctan \frac{\tilde{x}(t)}{x(t)}$.

The instantaneous frequency if $(t)$ can be expressed as the first derivative of the instantaneous phase (for a detailed discussion see Boashash, 1992a, b):

if $(t)=\frac{1}{2 \pi} \frac{d \omega(t)}{d t}$.

Figure 1 demonstrates the extraction of time-varying frequency information from a simple phase modulated sinusoidal signal.

\subsubsection{Adaptive Template Method (ATM)}

The Hilbert transform reacts sensitively on irregularities. Therefore the signal to noise ratio of the input signal has to be improved. This can be done with a newly developed approach, called Adaptive Template Method (ATM). The basic idea is to extract first a characteristic waveform, the template, which describes the typical grey-value variation transverse to the investigated laminae. In a second step, this template is used for a comparison with the actual grey-scale data. The generated signal shows maxima at positions, where the template optimally matches the original signal.

Annual grey-scale waves differ in terms of length, shape, and contrast. To obtain a characteristic waveform, several annual layers should be collected and averaged. ATM detects waves by searching for local maxima or minima of the initial grey-scale sequence. A certain predefined number of pixels in the surrounding of the extrema are used to generate a new set of small time series te $(m, q)=\left\{g s\left(x_{n}-q\right), \ldots, g s\left(x_{n}+q\right)\right\}$, where $q$ is the half length of the window $w=2 q+1$, and $m$ an index of the extracted wave. The maximum or minimum is positioned at the centre of $t e(m, q)$. After standardizing the sub-samples $t e_{s t}(q, m)$, a characteristic waveform is generated by averaging:

$t e_{s t}(m, q)=\frac{t e(m, q)-\mu(t e(m, q))}{\sigma(t e(m, q))}$. 
$\mu(m, q)$ arithmetic mean of te $(m, q), \sigma(t e(m, q))$ standard deviation of $t e(m, q)$

$t e(q)=\frac{\sum_{m=0}^{M-1} t e_{s t}(m, q)}{M}$,

$M$ : number of sub-samples.

This algorithm is a kind of a "learning-process". The comparison of templates and grey-scale variation transverse to the layers is done by calculating the scalar product between them. To eliminate the influence of amplitudes, the product of absolute values of template and grey-scale sequence divides the scalar product:

$y\left(x_{n}\right.$, te $\left.(q)\right)=\operatorname{AT}\left(x_{n}\right.$, te $\left.(q)\right)=\frac{\left\langle\text { te }(q) s\left(x_{n}\right)\right\rangle}{|t e(q)|\left|s\left(x_{n}\right)\right|}$.

High accordance between grey-values and template leads to results near 1.0, bad accordance generates values near 0.0. The created time-series is called the AT transform. It is smoother than the initial data sequence. High frequency noise and local trend effects are suppressed. Figure 2 shows an example for a typical greyscale data set and its AT transform. Afterwards, the AT transform can be analysed with traditional spectral methods. To extract instantaneous phase information, the Hilbert transform is applied on the AT transform.

The idea of the ATM is similar to basic algorithms of the wavelet transform. The main difference is that the actual shape of the template is adapted to the individual time series, so that the template optimally matches the properties of each signal. Actually, in some cases the template and the complex Morlet wavelet show a comparable appearance. Then, the results of both methods are also similar. But in general, the ATM due its adaptive origin seems to be the more appropriate method to preprocess noisy, frequency-modulated time series.

\section{Evaluation}

\subsection{Artificial data}

The performance and robustness of automated layerdetection methods should be tested under different conditions. Therefore, we created a simple, one-dimensional greyvalue model of annually accumulated biogenic sediments. This model supplies artificial data sets with various, adjustable characteristics.

To generate a synthetic signal, we used Eq. (1) with a base period of 10 units per year (sampling rate: $\Delta x=0.1$ ). Phase modulates with periods of $6.7,7.7,11$ and 16 years. These are common climate-driven periods, found in several paleoclimate studies (Ripepe et al., 1991; White et al., 1986). The strength of periodicities is unknown, so all get the same modulation index.

$s_{x}=\sin \left(2 \pi x+3 \sin \left(\frac{2}{16} \pi x\right)+3 \sin \left(\frac{2}{11} \pi x\right)+\right.$
Table 1. Results of tests on efficiency of methods

\begin{tabular}{lcccc}
\hline & $\begin{array}{c}\text { Differential } \\
\text { Filters } \\
\text { Method }\end{array}$ & $\begin{array}{c}\text { Moving } \\
\text { Average }\end{array}$ & $\begin{array}{c}\text { Hilbert } \\
\text { transform } \\
\text { Method }\end{array}$ & $\begin{array}{c}\text { Adaptive } \\
\text { Template }\end{array}$ \\
\hline $\begin{array}{l}\text { Amplitude } \\
\text { modulation }\end{array}$ & - & - & + & ++ \\
$\begin{array}{l}\text { Phase } \\
\text { modulation } \\
\text { Visible } \\
\text { disturbances }\end{array}$ & + & + & - & ++ \\
$\begin{array}{l}\text { Baruth } \\
\text { data }\end{array}$ & - & - & + & + \\
\hline
\end{tabular}

$$
\left.3 \sin \left(\frac{2}{7.7} \pi x\right)+3 \sin \frac{2}{6.7} \pi x\right)
$$

sampling rate: $\Delta x=0.1$, number of samples: $N=20000$.

For the creation of disperse disturbances, random numbers are added to phase or amplitude. The maximum size of random numbers is variable and depends on the desired disturbance strength. For the tests they increase in the scheme: $0 \%$, $10 \%, 30 \%, 50 \%, 70 \%, 90 \%$, and $100 \%$. Visible irregularities of different classes interrupt the regular sedimentation. They are modelled as constant grey-values, for instance dark greyvalues for drill core cracks or light colours for siderites and ash-fall layers. They range between $0 \%$ and $16.5 \%$. A selection of resulting periodograms can be seen in Fig. 3. Table 1 summarizes the performance of the algorithms.

Irregularities of amplitude modulation appear in various contexts. They occur as arbitrary differences in contrast, or, as an intra-annual layering. Both Differential Filters and Moving Average Method react on irregularities of amplitude modulation extremely sensitive. Background noise amplifies very quickly, so that at a disturbance portion of $30 \%$ random peaks already hides main frequencies. The number of laminae increases, whereas average varve thicknesses decrease. The Hilbert transform exhibits a more robust behaviour. But for irregularity-values greater than $50 \%$, there are high spurious peaks. The Adaptive Template Method works up to a disturbance factor of $90 \%$ reliably and is therefore the best decision for the treatment of amplitude irregularities.

Perturbations of phase modulation arise from different layer-altering effects, like additional sediment supply or postsedimentary processes (e.g. compaction and dehydration). The Hilbert transform without preprocessing reacts on disturbances of phase modulation most sensitive. All main peaks of the periodogram can only be detected at $10 \%$ irregular influence. Both varve-counting methods (Differential Filter, Moving Average Method) work up to a disturbance degree of $30 \%$ without problems. The Adaptive Template Method again is the best choice for treating phase problems. Up to $50 \%$ irregularities it supplies reliable results.

All newly developed approaches can be easily affected by visible disturbances. Both varve-counting methods react 


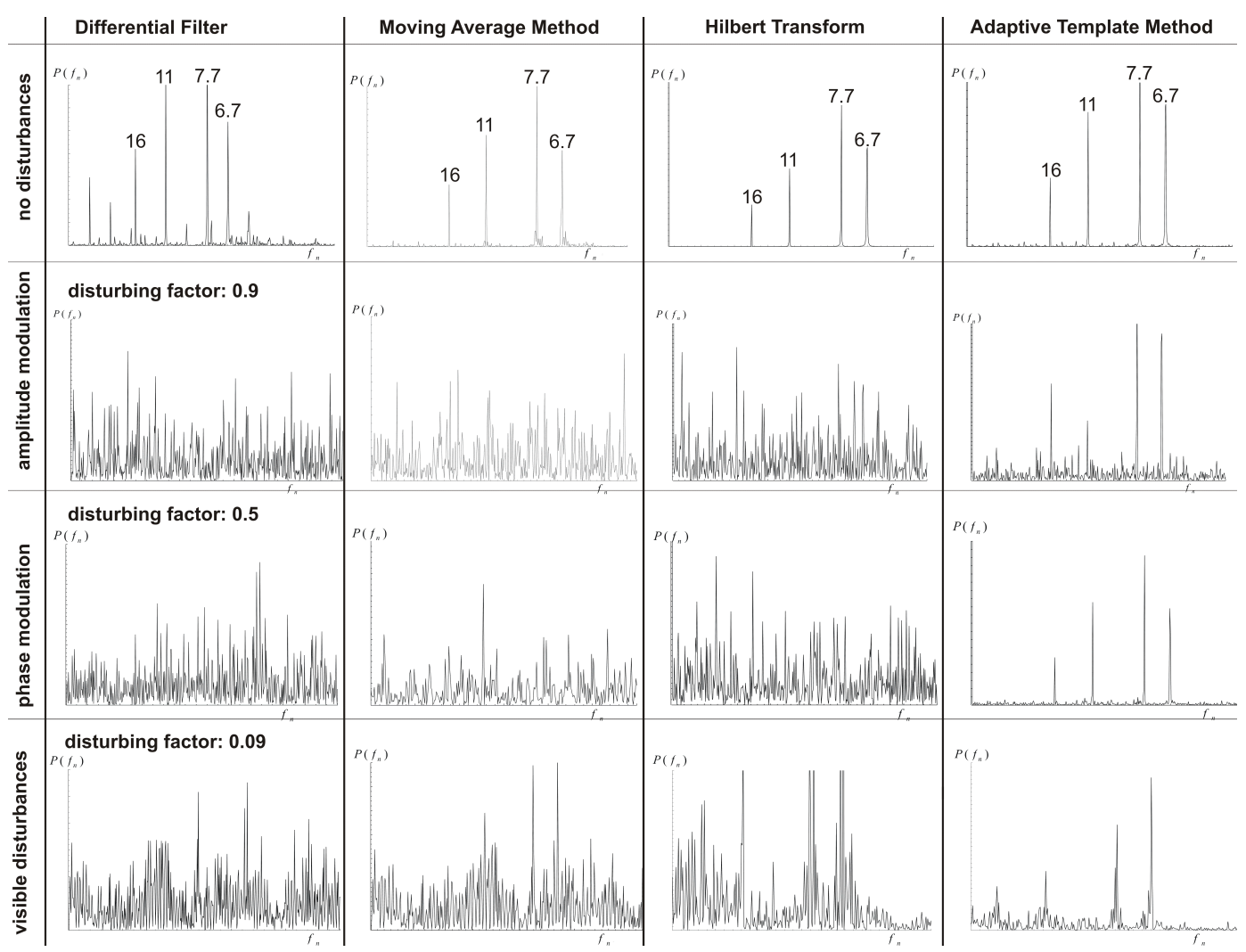

Fig. 3. Automated method evaluation with artificial signals. The upper row shows the final periodogram of the undisturbed signal after applying the automated data-processing methods. All algorithms detect the periods of 16, 11, 7.6, and 6.6 years. In the lower diagram adjustable perturbations (amplitude, phase, and visible disturbances) alter the original signal and thus the resulting representations in frequency space. Please note the different disturbing factors. Best results shows the Adaptive Template Method (ATM) in combination with the Hilbert transform.

extremely sensitive on this type of perturbations, and should not be used for such data. Up to an irregularity influence of $9 \%$ both Hilbert transform and the Adaptive Template Method work. But single peaks cannot be resolved clearly (effects of so called "peak doubling"), and background noise, especially at lower frequency parts, increases.

\subsection{Baruth Maar data}

The natural data set, we used for the evaluation of automated approaches, is based on algal laminites from drill cores of the Upper Oligocene Baruth Maar (Germany, Saxony). The lamination is due to light and dark layers. Light layers almost completely consist of central diatoms derived from algal blooms. Dark layers present the background sedimentation. They are built up of pennate diatom algae, but additionally contain chrysophyte cysts, clay and authigenic minerals (Goth, 2000; Goth et al., 2003). The intensity of algal blooms is climatically influenced. Therefore variation of lamina thicknesses indicates paleoclimatic changes.

The topmost $20 \mathrm{~m}$ of laminated sediments are nearly undisturbed. Based on an average sedimentation rate of $200 \mu \mathrm{m} / \mathrm{a}$, the data set contains a 100000 a lasting climate archive. For further investigations a $1 \mathrm{~cm}$ broad and
$20 \mathrm{~m}$ long drill core strip was scanned with high resolution $(1000 \mathrm{px} / \mathrm{cm})$. Subsequently several line scans were extracted along the core thereby excluding parts with visible disturbances. For further investigations we analysed a 395 a long sample from the centre of the grey-scale image and compared results of hand-digitisation and automated approaches in frequency space. Results are shown in Fig. 4.

Neither of the methods based on lamina detection (Differential Filters, Moving Average Method) is suitable for automated data processing. The lamina number generated by the Differential Filter method is even three times higher than that of hand-digitising. In comparison the lamina number derived by the Moving Average Method is with 387 varves nearly equal to the manual results. But signals differ both in time and frequency space.

Nearly every periodogram peak of the instantaneous frequency, derived by the Hilbert transform, reappears at the corresponding diagram of hand-digitised results. Certainly, several frequencies seem to be slightly displaced, and according to amplitudes, peaks are not in the right order. Another problem arises from spurious peaks. They appear in the HTperiodograms with partly high spectral power.

All peaks of the ATM-treated data sequence match to those of hand-digitising. But there remain differences in relative 


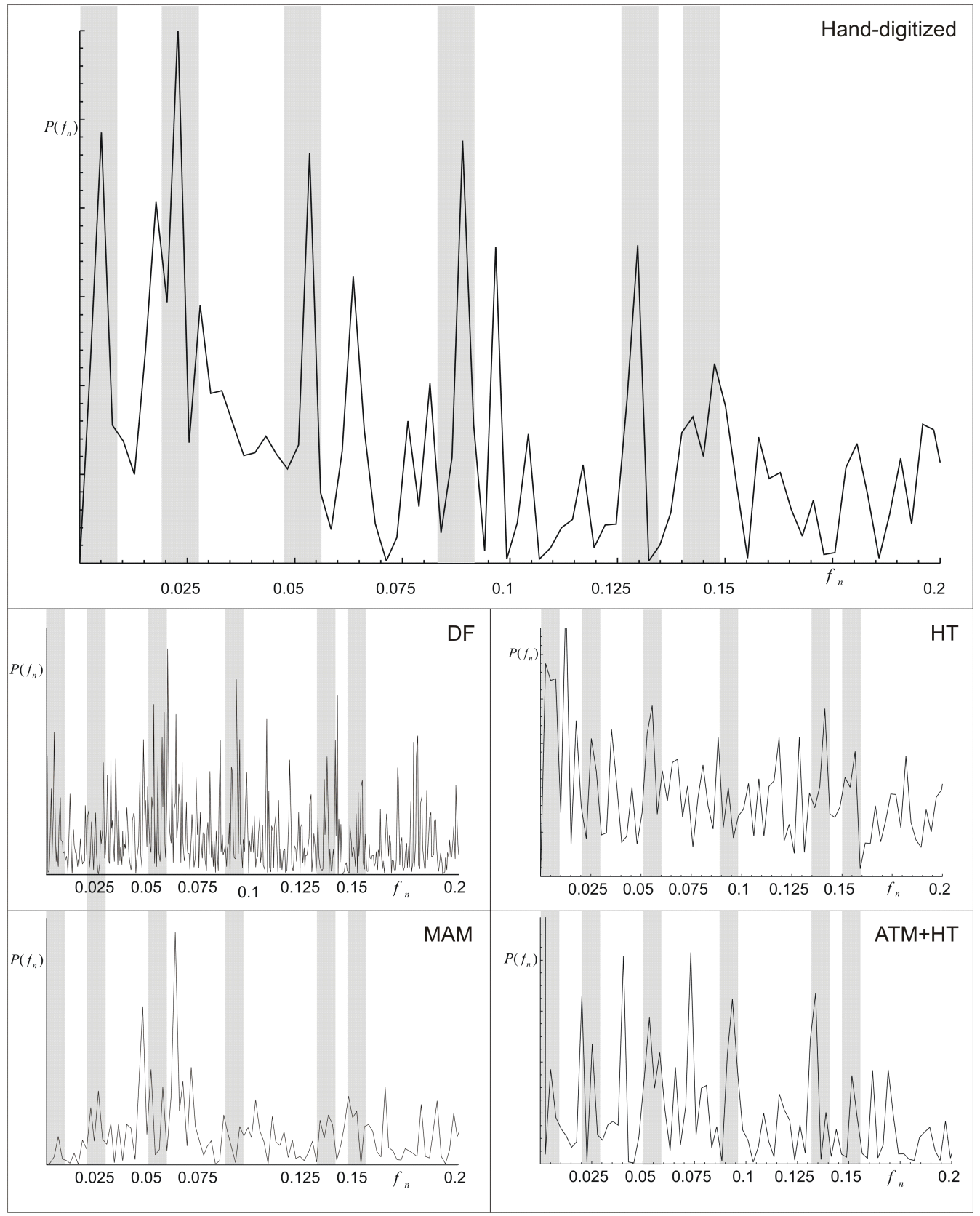

Fig. 4. Automated method evaluation with a natural data set from the Baruth Maar. The upper figure shows the resulting periodogram of a 395 a lasting hand-digitised core section. The final spectral representations after applying the different data-processing methods can be seen in the lower four diagrams (DF - Differential Filters, MAM - Moving Average Method, HT - Hilbert transform, ATM - Adaptive Template Method). The Adaptive Template Method in combination with the Hilbert transform shows the best results. All peaks of the ATM-treated data sequence match to those of hand-digitising.

spectral power values and problems with spurious frequencies and peak-doubling effects. Therefore, several templates with different window-lengths should be used for data processing. Oscillations that occur in all AT transforms can be interpreted as non-spurious.

The Adaptive Template Method turned out to be the best method of automated lamina-detection. A C++-program, called ATM-Explorer, uses this algorithm. For more information about the source code and the program please contact the author.

\section{Conclusions and future work}

Climatically induced fluctuations of the sedimentation rate of lake sediments can be approximated as phase modulated oscillation with a one-year base frequency. Regular lamina structures are interrupted and partly hidden by different disturbances. To extract computable data from the geological material, four new approaches for automated layer acquisition, based on 8 bit grey-scale curves, have been developed. They have been tested on artificial data and on the annually 
laminated Baruth Maar data. The Adaptive Template Method (ATM) gives the best results. It is an algorithm, which improves the signal to noise ratio and extracts, in combination with the Hilbert transform, instantaneous phase information.

Unlike manual or semi-manual approaches, ATM works quickly and objectively. It reacts robust against irregularities of the sedimentation rate (irregularities of phase modulation in a mathematical sense). Certainly, ATM is no panacea for strongly disturbed data sequences. Visible disturbances like drill core cracks or irregularly accumulated ash-fall layers, cause particular problems. In a subsequent spectral analysis, spurious frequencies may occur. Therefore it is recommended to use several ATM-results with different choices of parameters to identify and exclude spurious frequency peaks.

Until now, the Adaptive Template Method is still in development. At the moment, visible disturbances have to be removed manually before applying automated data processing algorithms. An automated detection, based on an adaptive learning process, would accelerate the signal processing procedure. Furthermore, it would be desirable to extend the algorithm for the treatment of two-dimensional data sets. This can be done with the help of some studies described in literature (Ripepe et al., 1991; D’ Arrigo et al., 1998; Katsuta et al., 2003). This step would improve the detection of intra-annual lamina thickness variations. The algorithms developed in this study can not only be used for annually laminated lake sediments but also for other types of time-dependent layering structures, like isotope distributions in ice-cores, or tree-rings in dendrochronology. They give a contribution to the quantification of geosciences and related disciplines.

Acknowledgements. We like to thank K. Goth (Sächsisches Landesamt für Umwelt und Geologie, Freiberg) for providing the Baruth Maar data sets and fruitful discussions about the genesis of annually laminated sediments. Furthermore, we would like to thank the two anonymous referees for their constructive comments.

Edited by: M. Thiel

Reviewed by: two referees

\section{References}

Anderson, R. Y. and Dean, W. E.: Lacustrine varve formation through time, Paleo. Paleo. Paleo., 62, 215-235, 1988.

Barnola, M. J., Raynaud, D., Korotkevich, Y. S., and Lorius, S.: Vostok ice core provides 160,000-year record of atmospheric $\mathrm{CO}_{2}$, Nature, 329, 408-414, 1987.

Boashash, B.: Estimating and interpreting the instantaneous frequency of a signal, part 1: fundamentals, Proc. of the IEEE, 80, 4, 538-568, 1992a.

Boashash, B.: Estimating and interpreting the instantaneous frequency of a signal, part 2: algorithms and applications, Proc. of the IEEE, 80, 4, 519-537, 1992b.

Bradbury, J. P. and Dean, W. E.: Elk Lake, Minnesota: Evidence for Rapid Climate Change in the north central United States, GSA Spec. Pap., 276, 1993.

Brauer, A., Hajdas, I., Negendank, J. F. W., Rein, B., Vos, H., and Zolitschka, B.: Warvenchronologie - Eine Methode zur abso- luten Datierung und Rekonstruktion kurzer und mittlerer solarer Periodizitäten, Geowiss., 12, 10-11, 325-332, 1994.

Brauer, A., Endres, C., and Negendank, J. F. W.: Lateglacial calendar year chronology based on annually laminated sediments from lake Meerfelder Maar, Germany, Quat. Internat., 61, 1725, 1999.

Briffa, K. R., Jones, P. D., Schweingruber, F., Karlen, W., and Shiyatov, S. G.: Tree-ring variables as proxy-climate indicators Problems with low-frequency signals, in: Climatic variations and forcing mechanisms of the last 2000 years, edited by Jones, P. D., Bradley, R. S., and Jouzel, J., NATO ASI series 1, Springer, London, Berlin, Heidelberg, New York, 11-41, 1996.

Brockwell, P. J. and Davis, R. A.: Time Series: Theory and Methods, Springer, New York, Berlin, Heidelberg, 1991.

Cole, J. E., Fairbanks, R. G., and Moore, M.: The spectrum of recent variability in the Southern Oscillation: results from the Tarawa Atoll coral, Science, 262, 1790-1793, 1992.

Cook, E. R., D'Arrigo, R. D., and Briffa, K. R.: A reconstruction of the North Atlantic Oscillation using tree-ring chronologies from North America and Europe, The Holocene, 8, 1, 9-17, 1998.

Cooper, M. C.: The use of digital image analysis in the study of laminated sediments, JPL, 19, 33-40, 1997.

Cramér, H. and Leadbetter, M. R.: Stationary and related stochastic processes, John Wiley \& Sons, New York, London, Sydney, 1964.

Crowley, K. D., Duchon, C. E., and Rhi, J.: Climate record in varved sediments of the Eocene Green River Formation, J. Geophys. Res., 91, D8, 8637-8647, 1986.

D’Argenio, B. D., Fischer, A. G., Richter, G. M., Longo, G., Pelosi, N., Molisso, F., and Duarte Morais, M. L.: Orbital cyclicity in the Eocene of Angola: visual and image-time-series analysis compared, Earth Plan. Sc. Lett., 160, 147-161, 1998.

Dean, W., Anderson, R., Bradbury, P. J., and Anderson, D.: A 1500year record of climatic and environmental change in Elk Lake, Clearwater County, Minnesota 1: Varve thicknesses and grayscale density, J. Poaleolimn., 27, 287-299, 2002.

DeShazer, D. J., Breban, R., Ott, E., and Roy, R.: Detecting phase synchronization in a chaotic laser array, Phys. Rev. Lett., 874, 044101, 2001.

Francus, P., Keimig, F., and Besonen, M.: An algorithm to aid varve counting and measurement from thin sections, J. Poaleolimn., 28, 283-286, 2002.

Gabor, D.: Theory of communication, J. Inst. Eelctr. Engin., 93, 3, 429-457, 1946.

Glenn, R. C. and Kelts, K.: Sedimentary rhythms in lake deposits, in: Cycles and events in stratigraphy, edited by Einsele G., Ricken, W., and Seilacher, A., Springer, Berlin, Heidelberg, New York, 188-221, 1991.

Goth, K.: Research drilling Baruth (Bth 98/1): The laminated sediments, Terra Nostra, 6, 156-159, 2000.

Goth, K., Suhr, P., and Schulz, R.: Zwei Forschungsbohrungen in das verdeckte Maar von Baruth (Sachsen), Zeitschrift f. Angewandte Geologie, 49, 1, 9-17, 2003.

Katsuta, N., Masao, T, Teruyuki, T., and Kumazawa, M.: Image processing to extract sequential profiles with high spatial resolution from $2 \mathrm{~d}$ map of deformed laminated patterns, Computers \& Geosciences, 29, 725-740, 2003.

Mari, J.-L., Glangeaud, F., and Coppens, F.: Signal processing for geologists and geophysicists, Éditions Technip, Paris, 1999.

Merkt, J. and Müller, H.: Varve chronology and palynology of the lateglacia in northwest germany from lacustrine sediments of Hämelsee in Lower Saxony, Quat Internat, 61, 41-59, 1999. 
O'Sullivan, P. E.: Annually-laminated lake sediments and the study of quaternary environmental changes - a review, Quat. Sci. Rev., 1, 245-313, 1983.

Pikovski, A., Rosenblum, M., and Kurths, J.: Synchronisation, Cambridge University Press, Cambridge, 432, 2001.

Quian Quiroga, R., Kraskov, A., Kreuz, T., and Grassberger, P.: Performance of different synchronization measures in real data: A case study on electroencephalographic signals, Phys. Rev. E., 65, 041903, 2002.

Ripepe, M., Roberts, L. T., and Fischer, A. G.: ENSO and sunspot cycles in varved Eocene oil shales from image analysis, J. Sed. Pet., 61, 7, 1155-1163, 1991.
Stuiver, M., Grootes, P. M., and Braziunas, T. F.: The GISP2 ${ }^{18} \mathrm{O}$ climate record of the past 16500 years and the role of the sun, ocean and volcanoes, Quat. Res., 44, 341-354, 1995.

White, J. W. C., Gorodetzky, D., Cook, E. R., and Barlow, L. K.: Frequency analysis of annually resolved, 700 year paleoclimate record from GISP2 ice core, in: Climatic variations and forcing mechanisms of the last 2000 years, edited by Jones, P. D., Bradley, R. S., and Jouzel, J., NATO ASI series 1, Springer, London, Berlin, Heidelberg, New York, 193-212, 1996.

Zolitschka, B.: Paläoklimatische Bedeutung laminierter Sedimente, Gebrüder Borntraeger, Berlin, Stuttgart, 1998. 